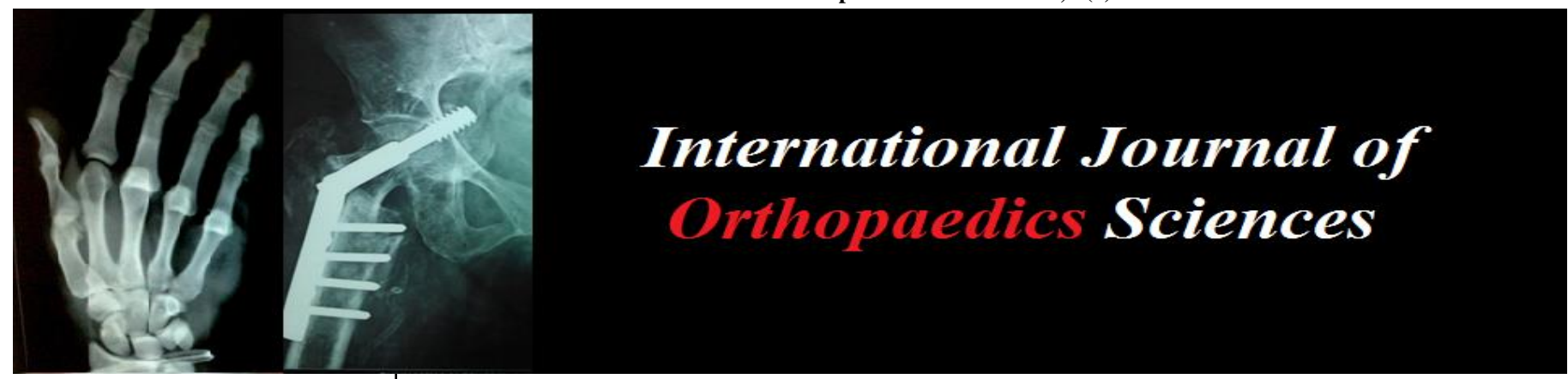

E-ISSN: 2395-1958

P-ISSN: 2706-6630

IJOS 2021; 7(2): 745-748

(C) 2021 IJOS

www.orthopaper.com

Received: 10-02-2021

Accepted: 12-03-2021

Dr. Deepak Singh Rajput Assistant Professor,

Department of Orthopaedics, MGM Medical College, Indore, Madhya Pradesh, India

Dr. Sharad Dubey

Resident, Department of Orthopaedics, MGM Medical

College, Indore, Madhya

Pradesh, India
Corresponding Author: Dr. Sharad Dubey Resident, Department of Orthopaedics, MGM Medical College, Indore, Madhya Pradesh, India

\section{Role of intra articular steroid injection in the management of adhesive capsulitis of shoulder joint}

\author{
Dr. Deepak Singh Rajput and Dr. Sharad Dubey
}

DOI: https://doi.org/10.22271/ortho.2021.v7.i2j.2699

\section{Abstract}

Adhesive capsulitis, is an idiopathic condition characterized by stiffness and pain in shoulder joint. Signs and symptoms typically begin gradually, worsen over time and then resolve, usually within one to three years. The prevalence found to be $3.06 \%$.

It is condition of painful shoulder with insidious onset with difficulty in sleeping on the affected side and in daily routine activities.

In this study we evaluate the efficacy of single intra-articular steroid injection in the patients with adhesive capsulitis. The study was conducted in twenty patients having established adhesive capsulitis of shoulder joint allocated for intra-articular injection of steroid in the shoulder through anterior approach. All the concerned patients assessed pre-operative and post-operative period by using DASH, VAS and $\mathrm{ROM}$ at regular intervals.

The study resulted in gross, constant, risk free and statistically significant improvement in pain in terms of DASH, VAS and functional ROM in patients with Adhesive Capsulitis.

Keywords: AC-adhesive capsulitis, DASH-Disability of arm, shoulder \& hand, VAS-visual analog score

\section{Introduction}

Adhesive capsulitis (AC), often referred to as Frozen Shoulder, is characterized by initially painful and later progressively restricted active and passive glenohumeral $(\mathrm{GH})$ joint range of motion with spontaneous complete or nearly-complete recovery over a varied period of time. Persistent shoulder pain is a very common problem in the community that often has multifactorial underlying pathologies. Spontaneous shoulder pain can result from bursitis, tendonitis, rotator cuff tear, A.C. impingement syndrome, Glenohumeral osteoarthritis and degenerative joint disease in which AC accounts for $6 \%$ of all shoulder pain.

Multiple intrinsic and extrinsic factors are thought to be responsible for disease progression. However, it is commonly believed that a combination of joint capsular contracture and fibrosis of rotator cuff interval, the subscapular \& coracohumeral ligament lead to movement restriction at Glenohumeral joint; in multiple planes.

It is very essential to diagnose the shoulder pain in the community to decrease the disease burden. There is no any gold standard clinical diagnostic test available for AC, so AC is therefore a diagnosis of exclusion \& must rely on accurate assessment of active and passive ROM. The globally accepted diagnostic criteria for AC is shoulder joint pain associated with restriction of active and passive movements of joint in at least two planes of joint range of motion; one being external rotation.

\section{Aims}

Aims of the study are to evaluate the role of Intra-articular injection of steroid in the management of Adhesive Capsulitis of shoulder joint and to analyse pain, ROM and activities of daily life using DASH and VAS scores.

\section{Materials and Methods}

We conducted the study in the Department of Orthopaedics and Traumatology at MGMMC and MYH Indore; between August 2019 to January 2021.

$$
\sim 745 \sim
$$


The study is based purely on clinical observations so no specific investigations were done for outcome analysis.

The study was conducted for total duration of 18 months with minimum follow up of 6 months.

\section{Inclusion criteria}

Age of patient $\rightarrow 18$ years.

Both genders were taken.

Clinical signs and symptoms of adhesive capsulitis with pain and restricted range of motion at shoulder joint.

Subjects with idiopathic Adhesive Capsulitis were taken.

\section{Exclusion criteria}

Age of patient - less than 18 and more than 70 years.

Patients with diagnosed RA or Infective arthritis.

Post traumatic Shoulder joint pain and stiffness including intra articular fractures and shoulder dislocation.

Infections \& Tumors.

Patient not willing to participate.

\section{Methodology}

The prospective study is done at aforementioned centre; on patients arrived with shoulder pain. After establishment diagnosis of AC; patients were examined clinically \& for RBS as well. Patients are classified as per inclusion and exclusion criteria of the study.

After all primary arrangements done and with precautions, patient were taken for intraarticular steroid injection $(40 \mathrm{mg}$ Triamcenolone) under aseptic conditions.

Patients were followed up for 2 weeks, 4 weeks, 8 weeks and 6 months post injection. Pain assessment and scoring of results done using DASH, VAS and quantitative assessment of ROM.

\section{Statistical methods}

The functional outcome in form of data were organised, tabulated and analysed using appropriate statistical means. Quantitative data were expressed in terms of mean and qualitative data were expressed as percentage and frequency.

\section{Assessment tools}

DASH

VAS

Range of Motion at Shoulder joint. (External rotation, Abduction \& internal rotation).

\section{Results and Discussion}

The effect of steroid in Adhesive Capsulitis of shoulder joint was indirectly assessed by improvement in VAS and DASH scores. Several case reports and comparative studies have reported that with the use of intraarticular steroid injections there are improvements in sign and symptoms by pain scores, radiographic and histological evaluation without systemic side effects.

In our study 7 or $35 \%$ out of 20 patients were in the age group of $41-50$ years, 5 or $25 \%$ of patients were in $61-70$ years, 4 or $20 \%$ in $51-60$ years, 3 or $15 \%$ in $31-40$ years and 1 patient was in the age group of 18 to 30 . The prevalence of $\mathrm{AC}$ increased in people over 40 years of age. The mean age found in our study was 55 years. This may be due to age related changes in collagen and proteoglycans that decrease the tension of joint cartilage and also because of the reduced supply of nutrients to the cartilage.

In our study 9 male and 11 females were affected, the ratio being 1:1.22 Preponderance of female patients has been consistently seen in all studies which may be related to less cartilage volume and greater cartilage wear, differences in mechanical alignment, anatomic differences, and genetic and hormonal issues.

There were no complication during the course of study.

The duration of pain was 6-8 months in majority of patients. The average duration of pain was 7 months.

\section{ROM: (In degree)}

\section{External rotation}

The Mean pre injection was 11.0 degrees which increased to 18 degrees at 2 weeks, 25.0 degrees at 4 weeks, 35.0 degrees at 8 weeks and 40.0 degrees at final follow up of 6 months.

Hence, The Mean External Rotation increases from preprocedure 11.0 to 40.0 degrees at 6 months which showed a significant increase in External Rotation.

\section{Abduction}

The Mean Abduction pre injection was 40 degrees which increased to 65.0 degrees at 2 weeks, 84 degrees at 4 weeks, 110 degrees at 8 weeks and 145 degrees at final follow up of 6 months.

Hence Mean Abduction increase from pre-procedure 40 to 145 degrees at 6 months which showed excellent improvement in Abduction.

\section{Internal rotation}

The Mean Internal Rotation pre injection was 12 degrees which increased to 15.0 degrees at 2 weeks, 25 degrees at 4 weeks, 30.0 degrees at 8 weeks and 40.0 degrees at final follow up of 6 months.

Hence, Mean Internal Rotation increased from pre-procedure 12 to 40.0 degrees at 6 months which showed remarkable progress in Internal Rotation.

\section{DASH score}

DASH scoring was done to evaluate efficacy of intraarticular injection of steroid in $\mathrm{AC}$ of shoulder, before and after the procedure at 2 weeks, 4 weeks, 8 weeks and 6 months. In our study pre injection DASH score were 76 , which decrease to 60.6 in 2 weeks, 50.3 in 4 weeks, 34.2 in 8 weeks, and 20.2 at final follow up of 6 months.

Hence, Mean DASH Score decreased from pre-procedure 76 to 20.2 at 6 months which showed a significant decrease in DASH Score, and thus prove the effectiveness of the steroid therapy.

\section{VAS score}

The Visual Analog Scale is a validated, subjective measure for acute and chronic pain which is recorded by making a mark on a line that represents a continuum between 'no pain' and 'worst pain'.

To evaluate the efficacy of intraarticular injection of steroid in AC of shoulder we have periodically recorded VAS score before and after the procedure at 2 weeks, 4 weeks, 8 weeks and 6 months. The pre injection VAS score was 7.8 which decrease to 6.3 in 2 weeks, 5.1 in 4 weeks, 3.0 in 8 weeks and at final follow of 6 month it became 1.9 .

\section{Summary and Conclusion}

To summarize our prospective study of 20 cases of AC of shoulder that presented to the OPD or emergency to department of Orthopaedics and Traumatology, M.G.M Medical College and M.Y. Hospital, Indore between August 2019 to January 2021 and treated with single intra-articular 
injection of steroid.

Post procedure, the patients were followed regularly for a period of 6 months. In our study:

The mean age found was 55 years.

The incidence of AC of shoulder was higher in female patients as compared to male patients.

Right side was more frequently involved compared to left.

The rate of post injection complication is nil in this study probably due to local application of steroid.

The average duration of pain was 7 months.

The Mean External Rotation increase from pre-procedure 11.0 to 40.0 0degrees at 6 months which showed a significant increase in External Rotation.

The Mean Abduction increase from pre-procedure 40 to 145 degrees at 6 months which showed a significant increase in Abduction.

The Mean Internal Rotation increased from pre-procedure 12 to 40.0 degrees at 6 months which showed a significant increase in Internal Rotation.

In our study, Mean DASH Score decreased from preprocedure 76 to 20.2 at 6 months which showed a significant decrease in DASH Score. Steroid injections have shown effectiveness in providing pain relief in $\mathrm{AC}$ of shoulder joint patients. As a result total DASH score was significantly reduced at 6 months as compared to pre-procedure and thus prove the effectiveness of the steroid therapy.

In our study, a steroid injections have shown effectiveness in providing pain relief in $\mathrm{AC}$ of shoulder joint patients. As a result total VAS score was significantly reduced at 6 months as compared to pre-procedure and thus prove the effectiveness of the steroid therapy.

Two out of twenty patients did not have significant improvement at 6 months, both of them were diabetic and their RBS levels were found to be raised during subsequent follow ups. Any other reason except this was not found regarding failure of this procedure.

The clinical utility of use of single intraarticular steroid injection in subjective pain relief after 6 month of injection was proved.

Use of single steroid injection in the treatment of AC of shoulder has an advantage of being a simple procedure, taking only 30mins, financially at very low cost, requiring less surgical skill and hence a OPD/Minor procedure with negligible side effects and is more satisfying to the patient and helps treat unresponsive chronic pain and also delays progression of disease.
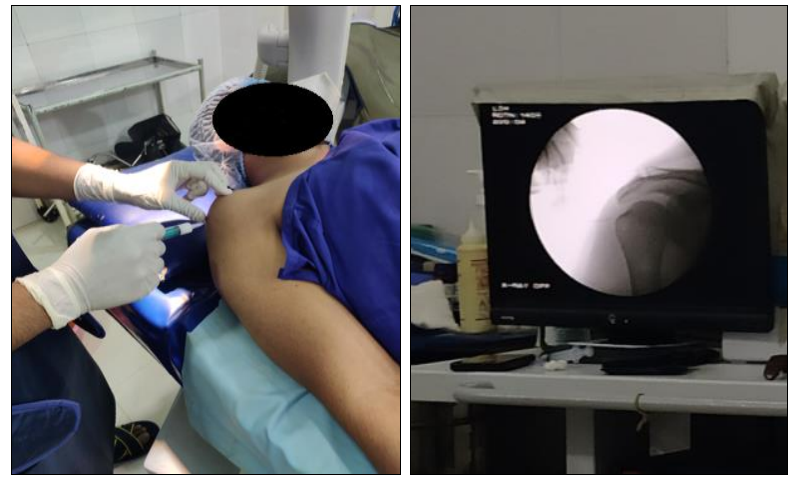

Fig 1: After patient preparation steroid being injected in shoulder joint
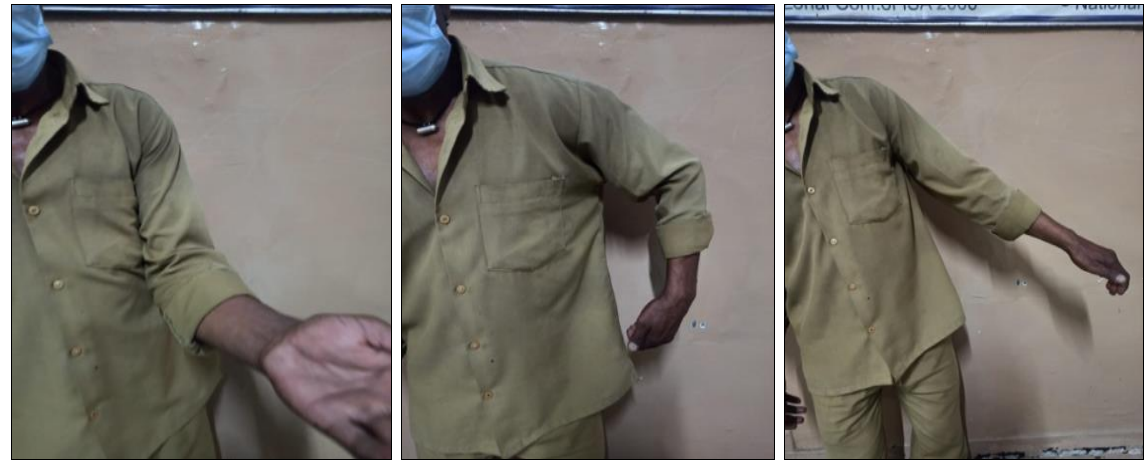

Fig 3: Pre injection clinical photos
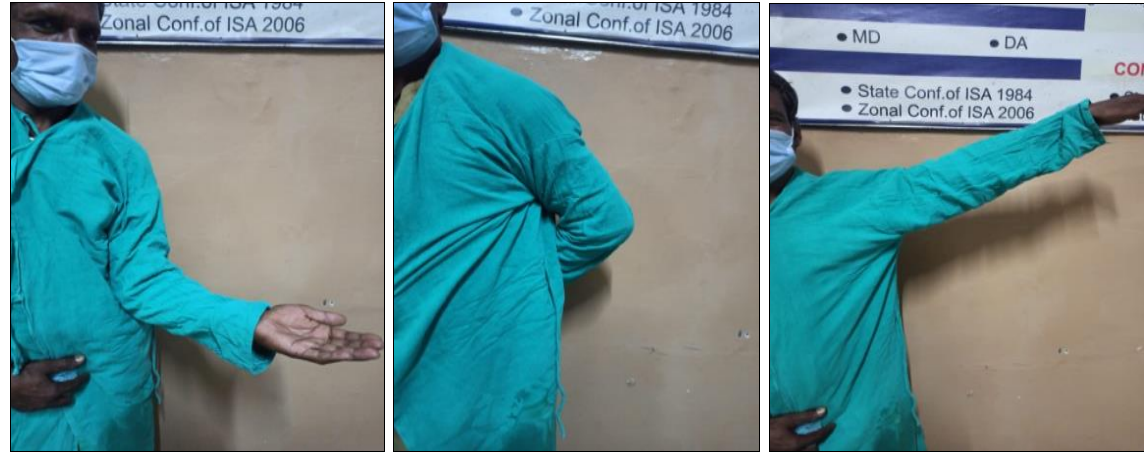

Fig 4: Post injection clinical photos at 6 months 


\section{References}

1. Uddin MM, Khan AA, Haig AJ, Uddin MK. Presentation of frozen shoulder among diabetic and non-diabetic patients. J ClinOrthop Trauma 2014;5(4):193-8.

2. Uppal HS, Evans JP, Smith C. Frozen shoulder: a systematic review of therapeutic options. World $\mathbf{J}$ Orthop 2015;6(2):263-8.

3. Manske RC, Prohaska D. Diagnosis and management of adhesive capsulitis. Curr Rev Musculoskelet Med 2008;1(3-4):180-9.

4. Robinson CM, Seah KT, Chee YH, Hindle P, Murray IR. Frozen shoulder. J Bone Joint Surg Br 2012;94(1):1-9.

5. Codman EA. The shoulder: rupture of the supraspinatus tendon and other lesions in or about the subacromial bursa. Malabar: Krieger Pub Co 1984.

6. Gautam VK, Verma S, Batra S, Bhatnagar N, Arora S. Platelet-rich plasma versus corticosteroid injection for recalcitrant lateral epicondylitis: clinical and Ultrasonographic evaluation. J Orthop Surg 2015;23(1):1-5

7. Daheshia M, Yao JQ. The interleukin 1beta pathway in the pathogenesis of osteoarthritis. J Rheumatol 2008;35:2306-2312.

8. Chen LX, Lin L, Wang HJ, Wei XL, Fu X, Zhang JY, Yu CL. Suppression of early experimental osteoarthritis by in vivo delivery of the adenoviral vector-mediated NFkappa Bp65-specific siRNA. Osteoarthr Cartil 2008;16:174-184.

9. Park KH, Na K. Effect of growth factors on chondrogenic differentiation of rabbit mesenchymal cells embedded in injectable hydrogels. J BiosciBioeng 2008;106:74-79.

10. Basak S, Kim H, Kearns JD, Tergaonkar V, O'Dea E, Werner SL et al. A fourth Ikappa B protein within the NF-kappa B signaling module. Cell 2007;128:369-381. 